ANNALES

POLONICI MATHEMATICI

$81.2(2003)$

\title{
The Kneser property for the abstract Cauchy problem
}

\author{
by Hernán R. Henríquez (Santiago) and \\ Genaro Castillo G. (Talca)
}

\begin{abstract}
We establish existence of mild solutions for the semilinear first order functional abstract Cauchy problem and we prove that the set of mild solutions of this problem is connected in the space of continuous functions.
\end{abstract}

1. Introduction. The purpose of this work is to show that the set formed by the mild solutions of a semilinear abstract Cauchy problem (ACP) of first order is connected in the space of continuous functions. This property is known in the literature as the Kneser property. We refer to [3] for the original result in the framework of differential equations and to [4] for a similar result for functional equations.

We start with an abstract statement of this property. In this statement we denote by $V_{\delta}(B)$ the $\delta$-neighborhood of a set $B$ in a metric space.

Lemma 1.1. Let $X, Y$ be metric spaces, $B$ a closed subset of $Y$, and $T: X \rightarrow Y$ a continuous function. Let $S=T^{-1}(B)$ and assume that there is a compact set $K \subseteq X$ such that for each $\varepsilon>0$ there is a set $K_{\varepsilon} \subseteq K$ with the following properties:

(i) the sets $K_{\varepsilon}$ are connected;

(ii) $d\left(x, K_{\varepsilon}\right)<\varepsilon$ for all $x \in S$;

(iii) $T\left(K_{\varepsilon}\right) \subseteq V_{\delta(\varepsilon)}(B)$, where $\delta(\varepsilon) \rightarrow 0$ as $\varepsilon \rightarrow 0$.

Then $S$ is connected.

Proof. Assume that $S$ is not connected. Then there exist nonempty disjoint closed sets $F_{1}$ and $F_{2}$ in $S$ such that $S=F_{1} \cup F_{2}$. This implies that both $F_{1}$ and $F_{2}$ are closed in $X$ and $d\left(F_{1}, F_{2}\right)=\eta>0$. Let $U$ be the $\eta / 2$-neighborhood of $F_{1}$. It is clear that $K_{\varepsilon} \cap U \neq \emptyset$ and $K_{\varepsilon} \cap(X \backslash \bar{U}) \neq \emptyset$ for each $0<\varepsilon<\eta / 2$. Since $K_{\varepsilon}$ is connected, it follows that $\operatorname{Fr}(U) \cap K_{\varepsilon} \neq \emptyset$.

2000 Mathematics Subject Classification: 47D06, 34G20.

Key words and phrases: abstract Cauchy problem; semigroup of operators.

Research of H. R. Henríquez was supported by DICYT-USACH, Project 04-0133HM and FONDECYT, Project 1020259. 
Thus, we can choose $x_{n} \in \operatorname{Fr}(U) \cap K_{n}$, where $K_{n}=K_{1 / n}$ for $n \in \mathbb{N}, n \geq N$. Since $K_{n} \subseteq K$, there is a subsequence, which we denote again by $\left(x_{n}\right)$, such that $x_{n} \rightarrow x$ as $n \rightarrow \infty$. Clearly, $x \in \operatorname{Fr}(U)$ and because $T$ is continuous, $T\left(x_{n}\right) \rightarrow T(x)$ as $n \rightarrow \infty$. Since $d\left(T\left(x_{n}\right), B\right) \rightarrow 0$ as $n \rightarrow \infty$, it follows that $d(T(x), B)=0$, which in turn implies that $T(x) \in B$ and $x \in S$. Consequently, $x \in \operatorname{Fr}(U) \cap S$, which is absurd by the construction of $U$.

In the next section we apply this result to the set formed by the solutions of a certain integral equation. To this end, the following version of Lemma 1.1 is more convenient. From now on, $X$ denotes a Banach space, $I \subseteq \mathbb{R}$ is a compact interval and $C(I ; X)$ stands for the space of continuous functions from $I$ into $X$ endowed with the norm of uniform convergence.

Corollary 1.1. Let $\mathcal{T}: C(I ; X) \rightarrow C(I ; X)$ be continuous and $S$ the set of fixed points of $\mathcal{T}$. Assume that there is a compact set $K \subseteq C(I ; X)$ and that for each $\varepsilon>0$ there is a set $K_{\varepsilon} \subseteq K$ with the following properties:

(i) the sets $K_{\varepsilon}$ are connected;

(ii) $d\left(x, K_{\varepsilon}\right)<\varepsilon$ for all $x \in S$;

(iii) $\|y-\mathcal{T} y\|_{\infty}<\delta(\varepsilon)$ for all $y \in K_{\varepsilon}$, where $\delta(\varepsilon) \rightarrow 0$ as $\varepsilon \rightarrow 0$.

Then $S$ is connected.

Another abstract version of the Kneser property, which is known as the Krasnosel'skiı-Perov theorem (see [9]), is obtained using degree theory.

We denote by $\mathcal{L}(X)$ the Banach space of bounded linear operators from $X$ into $X$.

2. First order abstract Cauchy problem. In this section we assume that $A$ is the infinitesimal generator of a strongly continuous semigroup of bounded linear operators $T(t)$ on $X$. We refer the reader to $[2,7]$ for the theory of strongly continuous semigroup and the associated ACP. We only mention here that every strongly continuous semigroup is uniformly bounded on bounded intervals. Moreover, a semigroup $T(\cdot)$ is called compact when the operators $T(t)$ are compact for all $t>0$.

The existence of solutions of the first order abstract Cauchy problem

$$
\begin{aligned}
x^{\prime}(t) & =A x(t)+h(t), \quad t \geq 0, \\
x(0) & =x_{0},
\end{aligned}
$$

where $h:[0, \infty) \rightarrow X$ is a locally integrable function, has been treated in several works. We only mention $[2,7]$ and the references therein. Similarly, the existence of solutions of the semilinear abstract Cauchy problem has 
been discussed in $[1,6]$. We only recall here that the function

$$
x(t)=T(t) x_{0}+\int_{0}^{t} T(t-s) h(s) d s, \quad t \geq 0,
$$

is called a mild solution of (2.1)-(2.2).

Our aim in this section is to establish a Kneser's type property for the solutions of the functional semilinear first order ACP

$$
x^{\prime}(t)=A x(t)+f(t, x(a(t))), \quad 0 \leq t \leq \tau,
$$

with initial condition (2.2). We abbreviate the notation by writing $I=[0, \tau]$ and we let $M$ indicate a constant such that $\|T(t)\| \leq M$ for all $0 \leq t \leq \tau$.

We begin with a result that ensures existence of solutions under quite general hypotheses which are suitable for our purposes. To study this initial value problem we always assume that the following conditions are fulfilled:

Assumption A. (a) The function $a: I \rightarrow I$ is continuous.

(b) The function $f: I \times X \rightarrow X$ satisfies the following Carathéodory conditions:

(i) $f(t, \cdot): X \rightarrow X$ is continuous for a.e. $t \in I$;

(ii) for each $x \in X$, the function $f(\cdot, x): I \rightarrow X$ is strongly measurable.

The expression (2.3) motivates the following concept of mild solution.

Definition 1. We say that a function $x: I \rightarrow X$ is a mild solution of the problem $(2.4),(2.2)$ if $x$ is a continuous function that satisfies the integral equation

$$
x(t)=T(t) x_{0}+\int_{0}^{t} T(t-s) f(s, x(a(s))) d s .
$$

In what follows we denote by $B_{R}$ the closed ball with center at 0 and radius $R$ in an appropriate space. Moreover, we denote by $\overline{\mathrm{co}}(B)$ the closed convex hull of a set $B$.

THEOREM 2.1. Assume that Assumption A and the following conditions hold:

(H-1) For each $R>0$ there is a positive integrable function $\gamma_{R} \in \mathcal{L}^{1}(I)$ such that $\sup \{\|f(t, x)\|:\|x\| \leq R\} \leq \gamma_{R}(t)$ for a.e. $t \in I$.

(H-2) For each $0<t \leq \tau$ and $R \geq 0$ the set $\{T(t) f(s, x): 0 \leq$ $s \leq \tau,\|x\| \leq R\}$ is relatively compact.

(H-3) $\quad \liminf _{R \rightarrow \infty} \frac{M}{R} \int_{0}^{\tau} \gamma_{R}(s) d s<1$. 
Then there is a mild solution of (2.4),(2.2). Furthermore, if the following condition is fulfilled:

$$
\limsup _{R \rightarrow \infty} \frac{M}{R} \int_{0}^{\tau} \gamma_{R}(s) d s<1,
$$

then the set $\mathcal{S}$ of mild solutions of $(2.4),(2.2)$ is compact in $C(I ; X)$.

Proof. Let $\mathcal{T}: C(I ; X) \rightarrow C(I ; X)$ be the map defined by

$$
\mathcal{T}(x)(t)=T(t) x_{0}+\int_{0}^{t} T(t-s) f(s, x(a(s))) d s .
$$

Clearly $\mathcal{T}$ is well defined and the Lebesgue dominated convergence theorem implies that $\mathcal{T}$ is continuous. We claim that there exists $n \in \mathbb{N}$ such that $\mathcal{T}: B_{n} \rightarrow B_{n}$. In fact, otherwise we can select a sequence $\left(x_{k}\right)_{k \in \mathbb{N}}$ in $C(I ; X)$ such that $\left\|x_{k}\right\|_{\infty} \leq k$ and $\left\|\mathcal{T}\left(x_{k}\right)\right\|_{\infty}>k$ for every $k \in \mathbb{N}$. Consequently, for $k \in \mathbb{N}$ from (2.6) we see that

$$
k<\left\|\mathcal{T}\left(x_{k}\right)\right\|_{\infty} \leq M\left\|x_{0}\right\|+M \int_{0}^{\tau} \gamma_{k}(s) d s,
$$

which yields

$$
1 \leq \liminf _{k \rightarrow \infty} \frac{M}{k} \int_{0}^{\tau} \gamma_{k}(s) d s,
$$

and this inequality contradicts $(\mathrm{H}-3)$.

Next, using (H-1) and (H-2), we establish that $\mathcal{T}$ is completely continuous. Since $\mathcal{T}$ is continuous it remains to prove that $\mathcal{T}$ takes bounded sets into relatively compact sets in $C(I ; X)$. From the Ascoli-Arzelà theorem it is sufficient to prove that for each $R \geq 0$ the set $\left\{\mathcal{T}_{0}(x)(t):\|x\|_{\infty} \leq R\right\}$ is relatively compact in $X$ for all $0 \leq t \leq \tau$ and that the set $\left\{\mathcal{T}_{0}(x):\|x\|_{\infty} \leq R\right\}$ is equicontinuous, where

$$
\mathcal{T}_{0}(x)(t)=\int_{0}^{t} T(t-s) f(s, x(a(s))) d s .
$$

We begin by establishing the first assertion. Let $t>0$ and take $\varepsilon>0$ small enough. We can write

$$
\begin{aligned}
\mathcal{T}_{0}(x)(t)= & \int_{0}^{t-\varepsilon} T(t-s) f(s, x(a(s))) d s+\int_{t-\varepsilon}^{t} T(t-s) f(s, x(a(s))) d s \\
= & \int_{0}^{t-\varepsilon} T(t-\varepsilon-s) T(\varepsilon) f(s, x(a(s))) d s \\
& +\int_{t-\varepsilon}^{t} T(t-s) f(s, x(a(s))) d s .
\end{aligned}
$$


The mean value theorem for the Bochner integral ([5]) shows that

$$
\begin{aligned}
& \int_{0}^{t-\varepsilon} T(t-\varepsilon-s) T(\varepsilon) f(s, x(a(s))) d s \\
& \quad \in(t-\varepsilon) \overline{c o}\{T(t-\varepsilon-s) T(\varepsilon) f(s, x):\|x\| \leq R, 0 \leq s \leq t-\varepsilon\} .
\end{aligned}
$$

By $(\mathrm{H}-2)$ the set on the right hand side is compact so that the first term on the right hand side of (2.7) is included in a compact set which does not depend on the function $x(\cdot)$.

On the other hand, for the second term on the right hand side of (2.7) we obtain the estimate

$$
\left\|\int_{t-\varepsilon}^{t} T(t-s) f(s, x(a(s))) d s\right\| \leq M \int_{t-\varepsilon}^{t} \gamma_{R}(s) d s,
$$

which shows that this term converges to zero as $\varepsilon \rightarrow 0$ since $\gamma_{R}$ is integrable. Hence the set $K(t)=\mathcal{T}_{0}\left(B_{R}\right)(t)$ is relatively compact.

To establish the second assertion we first observe that

$$
\begin{aligned}
\mathcal{T}_{0}(x)(t+h)-\mathcal{T}_{0}(x)(t)= & \int_{0}^{t+h} T(t+h-s) f(s, x(a(s))) d s \\
& -\int_{0}^{t} T(t-s) f(s, x(a(s))) d s \\
= & (T(h)-I) \int_{0}^{t} T(t-s) f(s, x(a(s))) d s \\
& +\int_{t}^{t+h} T(t+h-s) f(s, x(a(s))) d s .
\end{aligned}
$$

We observe that the first term on the right hand side is in $(T(h)-I) K(t)$ so it converges to zero as $h \rightarrow 0$, independently of $x(\cdot) \in B_{R}$. Similarly, the second term satisfies

$$
\left\|\int_{t}^{t+h} T(t+h-s) f(s, x(a(s))) d s\right\| \leq M \int_{t}^{t+h} \gamma_{R}(s) d s,
$$

which implies that it tends to 0 as $h \rightarrow 0$.

Applying now the fixed point theorem of Schauder we infer that $\mathcal{T}$ has a fixed point $x$ in $B_{n}$. Clearly $x$ is a mild solution of (2.4), (2.2). Moreover, the continuity of $\mathcal{T}$ implies that the set $\mathcal{S}$ of mild solutions is closed. 
On the other hand, if condition (H-4) holds then $\mathcal{S}$ is bounded. In fact, otherwise there exists a sequence $x_{k} \in \mathcal{S}$ such that $R_{k}=\left\|x_{k}\right\|_{\infty} \geq k$. Hence

$$
\left\|x_{k}(t)\right\|=\left\|\mathcal{T} x_{k}(t)\right\| \leq M\left\|x_{0}\right\|+M \int_{0}^{\tau} \gamma_{R_{k}}(s) d s,
$$

which yields

$$
1 \leq \limsup _{k \rightarrow \infty} \frac{M}{R_{k}} \int_{0}^{\tau} \gamma_{R_{k}}(s) d s
$$

but this is impossible by (H-4). Finally, using the fact that $\mathcal{T}$ is completely continuous we infer that $\mathcal{S}$ is compact. Thus the proof is complete.

REMARK. The semigroups that arise in applications are frequently compact. For this reason we point out that condition (H-2) holds when $T$ is compact and the function $f$ takes closed bounded sets into bounded sets.

Now we study the properties of $\mathcal{S}$. We assume that the conditions of Theorem 3.1 hold. We need the following property.

Lemma 2.1. Assume that $f$ is continuous and conditions (H-1) and (H-2) hold. Then for $R>0$ the set $\left\{\int_{0}^{t} T(t-s) f(s, y(s)) d s: 0 \leq t \leq \tau\right.$, $\left.y \in \mathcal{L}^{\infty}(I ; X),\|y\|_{\infty} \leq R\right\}$ is relatively compact.

Proof. Proceeding as in the proof of Theorem 2.1 we infer that the set

$$
V(t)=\left\{\int_{0}^{t} T(t-s) f(s, y(s)) d s: y \in \mathcal{L}^{\infty}(I ; X),\|y\|_{\infty} \leq R\right\}
$$

is relatively compact. We put $V=\bigcup_{0<t<\tau} V(t)$ and we claim that $V$ is relatively compact. We first observe that for each $\varepsilon>0$, there is $\delta>0$ such that

$$
V(t+h) \subseteq T(h) V(t)+B_{\varepsilon}, \quad|h| \leq \delta .
$$

In fact, choose $\delta>0$ such that $M \int_{t}^{t+h} \gamma_{R}(s) d s \leq \varepsilon$ for $h \leq \delta$. If $x \in V(t+h)$ then we can write

$$
\begin{aligned}
x & =\int_{0}^{t+h} T(t+h-s) f(s, y(s)) d s \\
& =T(h) \int_{0}^{t} T(t-s) f(s, y(s)) d s+\int_{t}^{t+h} T(t+h-s) f(s, y(s)) d s .
\end{aligned}
$$

Since the first term on the right hand side is in $T(h) V(t)$, and the norm of the second term is less than or equal to $M \int_{t}^{t+h} \gamma_{R}(s) d s$, we have established the claim. 
Now, assuming that $\delta=\tau / n$ for some $n \in \mathbb{N}$ large enough we have

$$
V \subseteq \bigcup_{i=0}^{n-1}\left(\bigcup_{0 \leq h \leq \delta} T(h) V(i \delta)+B_{\varepsilon}\right)=\bigcup_{0 \leq h \leq \delta} T(h)\left(\bigcup_{i=0}^{n-1} V(i \delta)\right)+B_{\varepsilon}
$$

which implies that $V$ is relatively compact.

Next we assume that the problem (2.4), (2.2) has mild solutions and we denote by $\mathcal{S}$ the set of those solutions. For $\mathcal{F} \subseteq C(I ; X)$ we denote by $\mathcal{F}(I)$ the set $\{f(t): f \in \mathcal{F}, t \in I\}$.

THEOREM 2.2. Assume that $f$ and a are continuous functions and that (H-1) and (H-2) are fulfilled. Suppose moreover that the following conditions hold:

(H-5) The set $\mathcal{S}$ is compact.

(H-6) For all $t \in I, a(t) \leq t$.

$$
3 M \liminf _{R \rightarrow \infty} \frac{1}{R} \int_{0}^{\tau} \gamma_{R}(s) d s<1 .
$$

Then $\mathcal{S}$ is connected.

Proof. Set $h(t)=T(t) x_{0}$. Applying (H-5) and (H-7) we can select a constant $R>0$ large enough such that $\|x\|_{\infty} \leq R$ for all $x \in \mathcal{S}$ and

$$
\|h\|_{\infty}+3 M \int_{0}^{\tau} \gamma_{R}(s) d s \leq R
$$

Let $V$ be the set constructed in the proof of Lemma 2.1. Without loss of generality we can assume that $V$ is absolutely convex. We set $U=2 V$, $U_{1}=3 V$ and $N_{1}=2 M \int_{0}^{\tau} \gamma_{R}(s) d s$.

We divide the proof into several steps.

STEP 1. For a partition $d$ of $I$ formed by the points $0=t_{0}<t_{1}<\ldots<$ $t_{n-1}<t_{n}=\tau$ we consider the function $z(\cdot)$ given by $z(0)=x_{0}$ and

$$
\begin{aligned}
z(t)= & h(t)+\sum_{k=1}^{i-1}\left(\int_{t_{k-1}}^{t_{k}} T(t-s) f\left(s, z\left(a\left(t_{k-1}\right)\right)\right) d s+\left(t_{k}-t_{k-1}\right) u_{k}\right) \\
& +\int_{t_{i-1}}^{t} T(t-s) f\left(s, z\left(a\left(t_{i-1}\right)\right)\right) d s+\left(t-t_{i-1}\right) u_{i}
\end{aligned}
$$

for $t_{i-1}<t \leq t_{i}$, where we choose $u_{k}$ so that $\sum_{k=1}^{i}\left(t_{k}-t_{k-1}\right) u_{k} \in U$ and $\left\|\sum_{k=1}^{i}\left(t_{k}-t_{k-1}\right) u_{k}\right\| \leq N_{1}$ for all $i=1, \ldots, n$. Clearly $z$ is a continuous function. Next, for fixed $z$ given by $(2.9)$ we denote by $y(\cdot)$ and $\varphi(\cdot)$ the step functions defined by $y(0)=x_{0}, \varphi(0)=u_{1}, y(t)=z\left(a\left(t_{k-1}\right)\right)$ and $\varphi(t)=u_{k}$ 
for $t_{k-1}<t \leq t_{k}$ and $k=1, \ldots, n$. Thus, we can rewrite the definition of $z$ as

$$
z(t)=h(t)+\int_{0}^{t} T(t-s) f(s, y(s)) d s+\int_{0}^{t} \varphi(s) d s .
$$

We shall show that $\|z(t)\| \leq R$ for $0 \leq t \leq \tau$, independently of the division $d$ and the choice of $u_{i}$. From (2.8) we easily obtain $\|z(t)\| \leq R$ for $0<t \leq t_{1}$. Assuming now that this inequality holds on $\left[0, t_{i-1}\right]$, we show it for $t_{i-1}<t \leq t_{i}$. In fact, since $\sum_{k=1}^{i-1}\left(t_{k}-t_{k-1}\right) u_{k}+\left(t-t_{i-1}\right) u_{i}$ is a convex combination of $\sum_{k=1}^{i-1}\left(t_{k}-t_{k-1}\right) u_{k}$ and $\sum_{k=1}^{i}\left(t_{k}-t_{k-1}\right) u_{k}$, from (2.9) we obtain

$$
\begin{aligned}
\|z(t)\| \leq & \|h(t)\|+\left\|\int_{0}^{t} T(t-s) f(s, y(s)) d s\right\| \\
& +\left\|\sum_{k=1}^{i-1}\left(t_{k}-t_{k-1}\right) u_{k}+\left(t-t_{i-1}\right) u_{i}\right\| \\
\leq & \|h\|_{\infty}+M \int_{0}^{\tau} \gamma_{R}(s) d s+2 M \int_{0}^{\tau} \gamma_{R}(s) d s,
\end{aligned}
$$

which establishes our assertion.

STEP 2. To simplify the construction we consider the points $t_{k}$ equally spaced with $\delta=t_{k}-t_{k-1}$ and, in addition to the conditions considered in Step 1, we suppose that

$$
\left\|\delta \sum_{k=i+1}^{j} u_{k}-[T((j-i) \delta)-I] \sum_{k=1}^{i} u_{k}\right\| \leq 2 M \int_{i \delta}^{j \delta} \gamma_{R}(s) d s
$$

for all $1 \leq i+1 \leq j \leq n$. We prove that the set $K$ formed by the functions $z$ defined by $(2.9)$ is relatively compact in $C(I ; X)$. Since $h$ is a fixed function, if we write $\widetilde{z}=z-h$ we must prove that $K_{0}=\{\widetilde{z}: z \in K\}$ is relatively compact.

From (2.9) and Lemma 2.1 it follows that

$$
\begin{aligned}
\widetilde{z}(t) & =\int_{0}^{t} T(t-s) f(s, y(s)) d s+\delta \sum_{k=1}^{i-1} u_{k}+\left(t-t_{i-1}\right) u_{i} \\
& \in V+U \subseteq U_{1}
\end{aligned}
$$

for every $z \in K$ and $t \in I$.

Now we prove that $K_{0}$ is equicontinuous. Let $0 \leq t^{\prime} \leq t \leq \tau$. From (2.9) we can write 


$$
\begin{aligned}
\widetilde{z}(t)-\widetilde{z}\left(t^{\prime}\right)= & \int_{0}^{t} T(t-s) f(s, y(s)) d s-\int_{0}^{t^{\prime}} T\left(t^{\prime}-s\right) f(s, y(s)) d s+\int_{t^{\prime}}^{t} \varphi(s) d s \\
= & {\left[T\left(t-t^{\prime}\right)-I\right] \int_{0}^{t^{\prime}} T\left(t^{\prime}-s\right) f(s, y(s)) d s } \\
& +\int_{t^{\prime}}^{t} T(t-s) f(s, y(s)) d s+\int_{t^{\prime}}^{t} \varphi(s) d s \\
= & {\left[T\left(t-t^{\prime}\right)-I\right]\left(\widetilde{z}\left(t^{\prime}\right)-\int_{0}^{t^{\prime}} \varphi(s) d s\right) } \\
& +\int_{t^{\prime}}^{t} T(t-s) f(s, y(s)) d s+\int_{t^{\prime}}^{t} \varphi(s) d s .
\end{aligned}
$$

Since $\widetilde{z}\left(t^{\prime}\right)-\int_{0}^{t^{\prime}} \varphi(s) d s \in V$ and $V$ is a compact set, it is sufficient to show that $\int_{t^{\prime}}^{t} \varphi(s) d s$ converges to zero as $t-t^{\prime} \rightarrow 0$, independently of the construction of $z$.

Since $U$ is compact and $\gamma_{R}$ is integrable, for every $\varepsilon>0$ there exists $\eta_{0}>0$ such that $\|(T(s)-I) u\| \leq \varepsilon / 2$ for all $0 \leq s \leq \eta_{0}$ and $u \in U$, and $2 M \int_{s^{\prime}}^{s} \gamma_{R}(s) d s \leq \varepsilon / 2$ for all $s^{\prime}, s \in I$ with $\left|s-s^{\prime}\right| \leq \eta_{0}$. We denote by $C_{1}$ the constant

$$
C_{1}=\sup \left\{s^{-1}\|(T(s)-I) u\|: \eta_{0} \leq s \leq \tau, u \in U\right\}
$$

and we take $0<\eta \leq \min \left\{\eta_{0}, \eta_{0} \varepsilon /\left(2 N_{1}\right), \varepsilon /\left(2 C_{1}\right)\right\}$.

First we assume that $t^{\prime}, t$ coincide with some points of the partition, say $t^{\prime}=t_{i}$ and $t=t_{j}$. In this case,

$$
\begin{aligned}
\int_{t^{\prime}}^{t} \varphi(s) d s & =\delta \sum_{k=i+1}^{j} u_{k} \\
= & \delta \sum_{k=i+1}^{j} u_{k}-(T((j-i) \delta)-I) \delta \sum_{k=1}^{i} u_{k} \\
& +(T((j-i) \delta)-I) \delta \sum_{k=1}^{i} u_{k}
\end{aligned}
$$

and applying (2.11) we obtain

$$
\left\|\int_{t^{\prime}}^{t} \varphi(s) d s\right\| \leq 2 M \int_{t^{\prime}}^{t} \gamma_{R}(s) d s+\left\|\left(T\left(t-t^{\prime}\right)-I\right) \delta \sum_{k=1}^{i} u_{k}\right\| .
$$

Consequently, since $\delta \sum_{k=1}^{i} u_{k} \in U$, if $t_{j}-t_{i} \leq \eta_{0}$ it follows that $\left\|\int_{t^{\prime}}^{t} \varphi(s) d s\right\|$ $\leq \varepsilon$. 
Let $t-t^{\prime} \leq \eta$. We analyze three possible situations with regard to the relative location of the points $t_{k}$. First, we assume that there is no $t_{k}$ between $t^{\prime}$ and $t$. Hence, there is an $i$ such that $t_{i}<t^{\prime}<t \leq t_{i+1}$ and $\int_{t^{\prime}}^{t} \varphi(s) d s=$ $\left(t-t^{\prime}\right) u_{i+1}$. From (2.11) we have

$$
\left\|u_{i+1}\right\| \leq \frac{2 M}{\delta} \int_{t_{i}}^{t_{i+1}} \gamma_{R}(s) d s+\left\|(T(\delta)-I) \sum_{k=1}^{i} u_{k}\right\| .
$$

Hence, if $\delta \leq \eta_{0}$, then since $t-t^{\prime} \leq \delta$, the above estimates imply that

$$
\left(t-t^{\prime}\right)\left\|u_{i+1}\right\| \leq \varepsilon / 2+\frac{t-t^{\prime}}{\delta}\left\|(T(\delta)-I) \delta \sum_{k=1}^{i} u_{k}\right\| \leq \varepsilon,
$$

while if $\delta \geq \eta_{0}$ we obtain

$$
\left(t-t^{\prime}\right)\left\|u_{i+1}\right\| \leq\left(t-t^{\prime}\right) \frac{N_{1}}{\delta}+\left(t-t^{\prime}\right) \frac{1}{\delta}\left\|(T(\delta)-I) \delta \sum_{k=1}^{i} u_{k}\right\| \leq \varepsilon,
$$

which establishes the assertion in this case.

Now we assume that there is an $i$ such that $t_{i-1}<t^{\prime}<t_{i}<t<t_{i+1}$. Then from our definitions it follows that

$$
\int_{t^{\prime}}^{t} \varphi(s) d s=\left(t_{i}-t^{\prime}\right) u_{i}+\left(t-t_{i}\right) u_{i+1}
$$

Since $t_{i}-t^{\prime}<t-t^{\prime}$ and $t-t_{i}<t-t^{\prime}$ we can argue as in the preceding case.

Finally, we assume that there is $i<j$ such that $t_{i-1}<t^{\prime}<t_{i}<t_{j}<t$ $<t_{j+1}$. Clearly,

$$
\left\|\phi(t)-\phi\left(t^{\prime}\right)\right\| \leq\left\|\phi(t)-\phi\left(t_{j}\right)\right\|+\left\|\phi\left(t_{j}\right)-\phi\left(t_{i}\right)\right\|+\left\|\phi\left(t_{i}\right)-\phi\left(t^{\prime}\right)\right\|
$$

where we have abbreviated $\phi(t)=\int_{0}^{t} \varphi(s) d s$. Since $t_{j}-t_{i} \leq t-t^{\prime}$, from our initial remark we obtain $\left\|\phi\left(t_{j}\right)-\phi\left(t_{i}\right)\right\| \leq \varepsilon$. Thus, this case is reduced to estimating the first and third terms on the right hand side of the above estimate. For the first term we observe that $\phi(t)$ is a convex combination of $\phi\left(t_{j}\right)$ and $\phi\left(t_{j+1}\right)$, so

$$
\left\|\phi(t)-\phi\left(t_{j}\right)\right\| \leq\left\|\phi\left(t_{j+1}\right)-\phi\left(t_{j}\right)\right\|
$$

and since $\delta=t_{j+1}-t_{j} \leq t-t^{\prime}$ we can repeat the previous argument. The third term is estimated similarly.

From the Ascoli-Arzelà theorem it follows that $K_{0}$ is relatively compact and hence so is $K=h+K_{0}$.

STEP 3. Let now $\varepsilon>0$ be fixed. Without loss of generality we also assume that $\varepsilon \leq \min \left\{\tau / 2,2 N_{1}\right\}$ and we take $\varepsilon_{1}=\varepsilon /(2 M \tau)$. Using the compactness of $\mathcal{S}$ and $K$, and the continuity of $f$ and $a$, we infer the existence 
of $0<\delta_{1} \leq \varepsilon$ such that

$$
\left\|f\left(s, x_{1}\right)-f\left(s, x_{2}\right)\right\| \leq \varepsilon_{1}
$$

for all $s \in I$ and for every $x_{1}, x_{2} \in(K \cup \mathcal{S})(I)$ such that $\left\|x_{1}-x_{2}\right\| \leq \delta_{1}$. Similarly, there is $\delta_{2}>0$ such that

$$
\|x(s)-x(t)\| \leq \delta_{1} / 4
$$

for all $x \in K \cup \mathcal{S}$ and $s, t \in I$ with $|t-s| \leq \delta_{2}$, and there is $\delta_{3}>0$ such that

$$
\|a(s)-a(t)\| \leq \delta_{2}
$$

whenever $|t-s| \leq \delta_{3}$. Now we choose $\delta=\tau / n \leq \min \left\{\delta_{1}, \delta_{2}, \delta_{3}, \delta_{1} /\left(2 M \varepsilon_{1}\right)\right\}$.

In what follows we consider the partition $d$ defined by $t_{i}=i \delta, i=$ $0, \ldots, n$. Let $K_{\varepsilon}$ be the set formed by the functions defined by (2.9) with $\left(u_{1}, \ldots, u_{n}\right) \in Z_{\varepsilon}$ where $Z_{\varepsilon}$ is the set of points $\left(u_{1}, \ldots, u_{n}\right) \in\left(2 \delta^{-1} U\right)^{n}$ that satisfy the following conditions:

$$
\begin{aligned}
& \text { (i) } \delta \sum_{k=1}^{i} u_{k} \in U ; \\
& \text { (ii) } \delta\left\|\sum_{k=1}^{i} u_{k}\right\| \leq M t_{i} \varepsilon_{1} ; \\
& \text { (iii) }\left\|\delta \sum_{k=i+1}^{j} u_{k}-\delta[T((j-i) \delta)-I] \sum_{k=1}^{i} u_{k}\right\| \leq 2 M \int_{t_{i}}^{t_{j}} \gamma_{R}(s) d s
\end{aligned}
$$

for all $i=1, \ldots, n$ and $j \geq i+1$.

We notice that condition (ii) implies that $\delta\left\|\sum_{k=1}^{i} u_{k}\right\| \leq N_{1}$. Next we establish some properties of $K_{\varepsilon}$.

STEP 4. The set $K_{\varepsilon}$ is connected. This is an easy consequence of the fact that the functions $z \in K_{\varepsilon}$ depend continuously on the choice of $\left(u_{1}, \ldots, u_{n}\right)$ $\in Z_{\varepsilon}$ and $Z_{\varepsilon}$ is convex by our construction.

STEP 5. In this step we show that the solutions of (2.5) can be approximated by elements in $K_{\varepsilon}$. Fix $x \in \mathcal{S}$. We proceed to define $z \in K_{\varepsilon}$ so that $\|x-z\|_{\infty} \leq \varepsilon$. We define $z(\cdot)$ inductively on the intervals $\left[t_{i-1}, t_{i}\right]$. To avoid some cumbersome expressions we set $p(t)=x(a(t))$ and $q(t)=z(a(t))$.

Let $i=1$. In this case $t_{1}=\delta$ and we take

$$
u_{1}=\frac{1}{t_{1}} \int_{0}^{t_{1}} T\left(t_{1}-s\right)[f(s, p(s))-f(s, p(0))] d s .
$$

It is clear from our construction that $u_{1} \in \delta^{-1} U$. Moreover, by (2.12)-(2.14) we have $\|f(s, p(s))-f(s, p(0))\| \leq \varepsilon_{1}$ for all $0 \leq s \leq \delta$, which implies that 
$\left\|u_{1}\right\| \leq M \varepsilon_{1}$. We define

$$
z(t)=h(t)+\int_{0}^{t} T(t-s) f(s, p(0)) d s+t u_{1}
$$

for $0 \leq t \leq t_{1}$. From this expression it follows that

$$
\begin{aligned}
z\left(t_{1}\right) & =h\left(t_{1}\right)+\int_{0}^{t_{1}} T\left(t_{1}-s\right) f(s, p(0)) d s+t_{1} u_{1} \\
& =h\left(t_{1}\right)+\int_{0}^{t_{1}} T\left(t_{1}-s\right) f(s, p(s)) d s=x\left(t_{1}\right) .
\end{aligned}
$$

Moreover, for $0<t \leq t_{1}$ we have

$$
\begin{aligned}
\|x(t)-z(t)\| & \leq\left\|\int_{0}^{t} T(t-s)[f(s, p(s))-f(s, p(0))] d s\right\|+t\left\|u_{1}\right\| \\
& \leq 2 M \varepsilon_{1} t \leq \delta_{1} / 2 .
\end{aligned}
$$

Proceeding by induction, we assume that we have selected elements $u_{k}$, $k=1, \ldots, i-1$, such that $\left(u_{1}, \ldots, u_{i-1}, 0, \ldots, 0\right) \in Z_{\varepsilon}$ and the function $z(t)$ given by $(2.9)$ for $t \in\left[0, t_{i-1}\right]$ satisfies $z\left(t_{k}\right)=x\left(t_{k}\right)$ and the estimate

$$
\|x(t)-z(t)\| \leq \delta_{1} / 2, \quad 0 \leq t \leq t_{i-1}
$$

We now define the function $z$ on $\left[t_{i-1}, t_{i}\right]$. We begin by selecting

$$
\begin{aligned}
u_{i}= & \frac{1}{\delta} \sum_{k=1}^{i-1} \int_{t_{k-1}}^{t_{k}}\left[T\left(t_{i}-s\right)-T\left(t_{i-1}-s\right)\right]\left[f(s, p(s))-f\left(s, q\left(t_{k-1}\right)\right)\right] d s \\
& +\frac{1}{\delta} \int_{t_{i-1}}^{t_{i}} T\left(t_{i}-s\right)\left[f(s, p(s))-f\left(s, q\left(t_{i-1}\right)\right)\right] d s .
\end{aligned}
$$

Utilizing the function $y(\cdot)$ defined previously we can write

$$
\begin{aligned}
u_{i}= & \frac{1}{\delta} \int_{0}^{t_{i}} T\left(t_{i}-s\right)[f(s, p(s))-f(s, y(s))] d s \\
& -\frac{1}{\delta} \int_{0}^{t_{i-1}} T\left(t_{i-1}-s\right)[f(s, p(s))-f(s, y(s))] d s .
\end{aligned}
$$

First we establish that $\left(u_{1}, \ldots, u_{i}, 0, \ldots, 0\right) \in Z_{\varepsilon}$. From the above expression it follows easily that $\delta u_{i} \in 2 U$ and

$$
\delta \sum_{k=1}^{i} u_{k}=\int_{0}^{t_{i}} T\left(t_{i}-s\right)[f(s, p(s))-f(s, y(s))] d s
$$


which implies that $\delta \sum_{k=1}^{i} u_{k} \in U$ and $\delta\left\|\sum_{k=1}^{i} u_{k}\right\| \leq N_{1}$. Moreover, for $m+1 \leq j \leq i$, we have

$$
\begin{aligned}
\delta \sum_{k=m+1}^{j} u_{k}= & \delta \sum_{k=1}^{j} u_{k}-\delta \sum_{k=1}^{m} u_{k} \\
= & \int_{0}^{t_{j}} T\left(t_{j}-s\right)[f(s, p(s))-f(s, y(s))] d s \\
& -\int_{0}^{t_{m}} T\left(t_{m}-s\right)[f(s, p(s))-f(s, y(s))] d s \\
= & {\left[T\left(t_{j}-t_{m}\right)-I\right] \int_{0}^{t_{m}} T\left(t_{m}-s\right)[f(s, p(s))-f(s, y(s))] d s } \\
& +\int_{t_{m}}^{t_{j}} T\left(t_{j}-s\right)[f(s, p(s))-f(s, y(s))] d s,
\end{aligned}
$$

which yields

$\delta \sum_{k=m+1}^{j} u_{k}-\left[T\left(t_{j}-t_{m}\right)-I\right] \delta \sum_{k=1}^{m} u_{k}=\int_{t_{m}}^{t_{j}} T\left(t_{j}-s\right)[f(s, p(s))-f(s, y(s))] d s$,

which in turn implies the estimate

$$
\left\|\delta \sum_{k=m+1}^{j} u_{k}-[T((j-m) \delta)-I] \delta \sum_{k=1}^{m} u_{k}\right\| \leq 2 M \int_{t_{m}}^{t_{j}} \gamma_{R}(s) d s .
$$

In addition, for $t_{i-1}<s \leq t_{i}$ we can write

$$
p(s)-y(s)=p(s)-p\left(t_{i-1}\right)+p\left(t_{i-1}\right)-q\left(t_{i-1}\right)+q\left(t_{i-1}\right)-y(s) .
$$

From (2.13) and (2.14) we have $\left\|p(s)-p\left(t_{i-1}\right)\right\| \leq \delta_{1} / 4$ and by induction $\left\|q\left(t_{i-1}\right)-y(s)\right\|=0$ and $\left\|p\left(t_{i-1}\right)-q\left(t_{i-1}\right)\right\| \leq \delta_{1} / 2$. Combining these with (2.12) we infer that $\delta\left\|\sum_{k=1}^{i} u_{k}\right\| \leq M t_{i} \varepsilon_{1}$, which completes the proof that $\left(u_{1}, \ldots, u_{i}, 0, \ldots, 0\right) \in Z_{\varepsilon}$.

Now we define $z(t)$ for $t_{i-1}<t \leq t_{i}$ by means of (2.9). Using this expression as well as the choice of $u_{k}, k=1, \ldots, i$, we infer that

$$
\begin{aligned}
x\left(t_{i}\right)-z\left(t_{i}\right)= & \int_{0}^{t_{i}} T\left(t_{i}-s\right)[f(s, p(s))-f(s, y(s))] d s-\delta \sum_{k=1}^{i} u_{k} \\
= & \int_{0}^{t_{i}} T\left(t_{i}-s\right)[f(s, p(s))-f(s, y(s))] d s \\
& -\int_{0}^{t_{i-1}} T\left(t_{i-1}-s\right)[f(s, p(s))-f(s, y(s))] d s-\delta u_{i}=0 .
\end{aligned}
$$


Moreover, from (2.13) and the choice of $\delta$ it follows that

$$
\|x(t)-z(t)\| \leq\left\|x(t)-x\left(t_{i-1}\right)\right\|+\left\|z(t)-z\left(t_{i-1}\right)\right\| \leq \delta_{1} / 2,
$$

which establishes the assertion.

STEP 6. In this step we prove that the elements of $K_{\varepsilon}$ are approximate solutions of (2.5). Specifically, we show that

$$
\left\|z(t)-h(t)-\int_{0}^{t} T(t-s) f(s, z(s)) d s\right\| \leq \varepsilon
$$

for all $t \in I$ and $z \in K_{\varepsilon}$. In fact, for $t_{i-1}<t \leq t_{i}$ using (2.9) we have

$$
\begin{aligned}
z(t)-h(t)-\int_{0}^{t} T(t-s) f(s, q(s)) d s= & \int_{0}^{t} T(t-s)[f(s, y(s))-f(s, q(s))] d s \\
& +\delta \sum_{k=1}^{i-1} u_{k}+\left(t-t_{i-1}\right) u_{i},
\end{aligned}
$$

and employing now (2.12) and the choice of $\delta$ we can establish the estimate

$$
\begin{aligned}
\| z(t)- & h(t)-\int_{0}^{t} T(t-s) f(s, q(s)) d s \| \\
\leq & \left\|\int_{0}^{t} T(t-s)[f(s, y(s))-f(s, q(s))] d s\right\|+\left\|\delta \sum_{k=1}^{i-1} u_{k}+\left(t-t_{i-1}\right) u_{i}\right\| \\
\leq & 2 M \tau \varepsilon_{1},
\end{aligned}
$$

which shows our assertion.

Finally, collecting Steps 1 to 6 and applying Corollary 1.1 we complete the proof.

When $a(t)=t$ we obtain the classical semilinear ACP

$$
x^{\prime}(t)=A x(t)+f(t, x(t)), \quad 0 \leq t \leq \tau .
$$

However, in this case we can establish the result without the stronger condition (H-7). From now on, we denote by $\mathcal{S}$ the set of mild solutions of (2.16), (2.2).

TheOrem 2.3. Assume that $f$ is continuous, conditions $(\mathrm{H}-1)-(\mathrm{H}-3)$ are fulfilled and $\mathcal{S}$ is compact. Then $\mathcal{S}$ is connected.

Proof. Since the argument is very similar to that used in the proof of the preceding theorem we only present the main aspects. Moreover, we keep the terminology introduced in the previous proof.

From Theorem 2.1 we know that $\mathcal{S}$ is nonempty. Since $\mathcal{S}(I)$ is compact we can take $N=\sup \{\|f(s, x)\|: s \in I, x \in \mathcal{S}(I)\}$. Applying (H-3) we infer 
that there exists $R>0$ such that $\|x\|_{\infty} \leq R$ for all $x \in \mathcal{S}$ and

$$
\|h\|_{\infty}+2 N M \tau+M \int_{0}^{\tau} \gamma_{R}(s) d s \leq R
$$

In this case we take as $U$ an absolutely convex set such that

$$
\tau \overline{\mathrm{co}}\{T(t) f(s, x): s, t \in I, x \in \mathcal{S}(I)\} \subseteq U .
$$

We define $z(\cdot)$ by $(2.9)$ where the elements $u_{k}$ are chosen so that

$$
\sum_{k=1}^{i}\left(t_{k}-t_{k-1}\right) u_{k} \in 2 U \text { and }\left\|\sum_{k=1}^{i}\left(t_{k}-t_{k-1}\right) u_{k}\right\| \leq 2 N M \tau .
$$

It is easy to see that $\|z(t)\| \leq R, t \in I$.

Defining $V, K_{0}, K$ as in the proof of Theorem 2.2 we find that $K$ is relatively compact.

Proceeding by induction we define $u_{i}$ by (2.15). In this case $p(s)=x(s)$, and for $t_{i-1}<s \leq t_{i}$, we obtain $y(s)=z\left(a\left(t_{i-1}\right)\right)=z\left(t_{i-1}\right)=x\left(t_{i-1}\right)$. This allows us to conclude the proof proceeding as in Theorem 2.2.

\section{References}

[1] T. Cazenave and A. Haraux, An Introduction to Semilinear Evolution Equations, Oxford Univ. Press, Oxford, 1998.

[2] K-J. Engel and R. Nagel, One-Parameter Semigroups for Linear Evolution Equations, Springer, New York, 2000.

[3] P. Hartman, Ordinary Differential Equations, Wiley, New York, 1964.

[4] T. Kaminogo, Kneser's property and boundary value problems for some retarded functional differential equations, Tohoku Math. J. 30 (1978), 471-486.

[5] C.-M. Marle, Mesures et Probabilités, Hermann, Paris, 1974.

[6] I. Miyadera, Nonlinear Semigroups, Amer. Math. Soc., Providence, 1992.

[7] A. Pazy, Semigroups of Linear Operators and Applications to Partial Differential Equations, Springer, New York, 1983.

[8] S. D. Zaidman, Abstract Differential Equations, Res. Notes in Math. 36, Pitman, San Francisco, 1979.

[9] E. Zeidler, Nonlinear Functional Analysis and its Applications, I, Springer, New York, 1986.

Departamento de Matemática

Universidad de Santiago

Casilla 307, Correo 2

Santiago, Chile

E-mail: hhenriqu@lauca.usach.cl
Departamento de Matemática Universidad de Talca Talca, Chile E-mail: gcastill@pehuenche.utalca.cl 